This paper was not presented at the conference:

Title: $\quad$ All-Fiber Laser Systems That Can Operate in Burst Mode

Authors: $\quad$ Denizhan Koray Kesim, Hamit Kalaycıoğlu, Önder Akçaalan, and Fatih

Event Name: 3D Image Acquisition and Display: Technology, Perception and Applications Year: $\quad 2016$ 


\title{
All-Fiber Laser Systems That Can Operate in Burst Mode
}

\author{
Denizhan Koray Kesim ${ }^{1, *}$, Hamit Kalaycığ̆lu², Önder Akçaalan ${ }^{2}$, F. Ömer Ilday ${ }^{1,2}$ \\ ${ }^{1}$ Department of Electrical and Electronics Engineering, Bilkent Ankara, 06800, Turkey ${ }^{2}$ Department of Physics, \\ Bilkent University, 06800 Ankara, Turkey \\ *:dkkesim@ee.bilkent.edu.tr
}

\begin{abstract}
Fiber lasers which operate in burst-mode where densely spaced pulses occur inside bursts repeated at much lower repetition rates can be valuable tool for sensing and imaging. We introduce such lasers and propose possible applications.
\end{abstract}

OCIS codes: $140.3510,320.5550$.

\section{Fiber Lasers}

Specially designed fibers can act as wave guide to laser light. Further, the amplification process can be done optically pumping active fibers.

Fibers, by nature, are quite flexible wave guides, except rod type fibers which are not in scope of this submission. They can be bent to certain degree even without losing polarization properties of the laser. The flexibility property of fibers allow them to be very compact. They can be fitted inside of a box much smaller than solid-state lasers with similar parameters. Furthermore, since the free space components are few and laser beam is mostly travelling inside a wave guide, little maintenance is necessary to operate fiber laser systems. Thanks to the recent developments, fiber lasers can go very high powers, which was limited before. Average power over $100 \mathrm{~W}$ is reported for burst-mode fiber laser with picosecond pulses [1].

\section{Burst Mode}

Traditionally, pulsed lasers operate in so-called uniform-mode where pulses are distributed equal in time. This mode practically limits the repetition rate for high energy pulses since the average power requirements raise which in turn produces the need for more complex laser systems. To overcome this, we send pulse bursts containing densely spaced pulses which in turn are repeated at much lower repetition rates, compared to pulse-to-pulse repetition rate inside individual bursts. This allows us to keep average power at sustainable levels. The potential of this mode of operation was first demonstrated by Marjoribanks and co-workers [2].

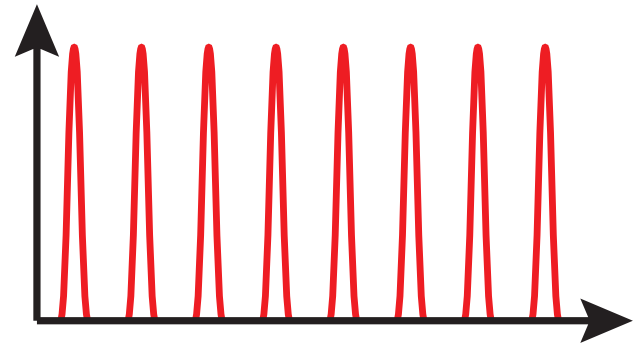

(a)

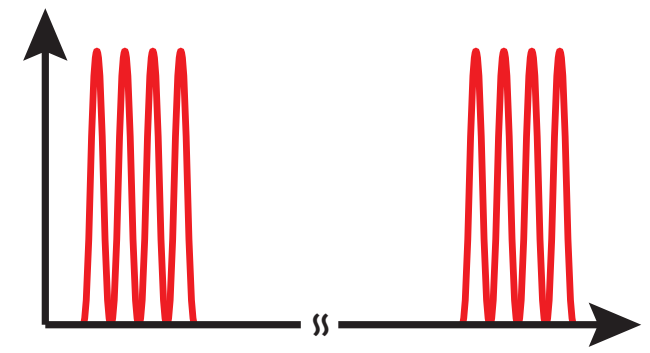

(b)

Fig. 1. Comparison of Burst-mode operation with uniform-mode operation. (a) The traditional uniform-mode where the pulses are separated equally. (b) Burst-mode where the pulses are packed closely and repeated at much lower repetition rates.

Burst-mode operation enables us to achieve great pulse energies at high repetition rates without the need to increase the laser complexity. The duty cycle is low in burst mode systems, and hence is the effective repetition rate of the 
system, which can be calculated as in Eq. 1 , where $f_{\text {burst }}$ is the burst repetition rate and $n_{\text {pulses }}$ is the number of pulses inside each burst.

$$
f_{\text {burst }} * n_{\text {pulses }}=f_{\text {effective }}
$$

This way, we can use all the advantages of a high repetition rate laser without the need to go to high powers. The effectiveness of burst-mode operation was demonstrated for material processing by enabling ablation cooled regime, which was discovered in our group in relation to laser material processing [3]. We have developed specialized fiber laser systems which use synchronized pulsed pumping to supress ASE and achieve high pulse energy at low burst repetition rates [4].

Furthermore, changing burst parameters such as number of pulses per burst and burst repetition rate can be seamless since bursts are generated by a fiber coupled AOM via pulse picking. An electronic circuit based on an FPGA synchronized with the seed signal from oscillator, that brings versatility to choose operation in burst-mode or in uniform-mode alternatively.

The pulses for bursts are picked by an AOM driven by FPGA which is triggered directly from laser oscillator. Thus, it is guaranteed to catch pulses properly with minimal loss.

\section{Current Systems}

These are the burst-mode fiber lasers systems that has been developed in our group.

\subsection{Compact Burst-Mode Fiber Laser System}

Originally developed for improving laser assisted cataract surgery by exploiting ablation cooled regime [5], it is a tabletop system that can be operated by medical doctors for further experiments. Yb fiber laset can generate repetition rate of $50 \mathrm{kHz}$ for bursts with intra-burst repetition rate of $91 \mathrm{MHz}$. Each burst can contain up to 48 pulses. Pulse durations are less than 300 femtosecond. The output can go up to $3 \mathrm{~W}$ which is distributed equally among pulses.

\subsection{High Repetition Rate Systems}

This $\mathrm{Yb}$ fiber laser system exceeds $\mathrm{GHz}$ repetition rate barrier by applying burst-mode operation [6]. Pulse-to-pulse repetition rate is increased to $3.5 \mathrm{GHz}$ from a $108 \mathrm{MHz}$ oscillator by using cascaded 50/50 couplers. Bursts have 215 $\mu \mathrm{J}$ energy distributed among 50 pulses which are repeated at $1 \mathrm{kHz}$ while keeping the average power as low as 200 $\mathrm{mW}$.

\section{LIDAR Application}

We propose burst-mode lasers for LIDAR sensing and imaging systems. Sensing in atmospheric medium through long distances requires high pulse energies for coherent detection. Only a fraction of light manages to return to be collected by sensors [7]. By using burst-mode operation, such high pulse energies are made possible for low average powers without giving up high repetition rate to get more information out of a single burst. This would reduce the cost of LIDAR systems for both building the light source and supplying power to the system.

\section{Conclusion}

Robustness and low maintenance makes fiber lasers desirable for various applications including imaging. The cost of building and maintaining them is lower compared to solid-state lasers. Recently, output of fiber lasers is increased dramatically so that even material processing applications are made possible. There are tunable fiber lasers that can be used for various applications.

Additionally, advantages of burst-mode operation has been recently illustrated experimentally. In this mode, pulses are gathered very closely in groups of bursts instead of distributing them equally temporally. It has been shown to achieve greater ablation rates compared to traditional uniform mode and employed to exploit ablation-cooled material processing. Burst-mode operation is especially useful for high repetition rate applications where average power was originally limiting.

Imaging technologies can benefit from either of them. Especially burst-mode operation is a promising tool for sensing. By using some pulse-to-pulse modulation, the possible applications can reach even further. We propose one possible application, LIDAR imaging and sensing, for burst-mode fiber lasers, though the application areas can be expanded. 


\section{References}

1. S. Yılmaz, P. Elahi, H. Kalaycıoğlu, and F. Ö. Ilday, "Amplified spontaneous emission in high-power burst mode fiber lasers," J. Opt. Soc. Am. B, vol. 32, no. 12, p. 2462, 2015.

2. M. Lapczyna, K. P. Chen, P. R. Herman, H. W. Tan, and R. S. Marjoribanks, "Ultra high repetition rate (133 MHz) laser ablation of aluminum with 1.2-ps pulses," Appl. Phys. A Mater. Sci. Process., vol. 69, no. 7, 1999.

3. F. Ö. Ilday, "Ultrafast burst-mode fiber lasers: Source development and material processing," Lasers and ElectroOptics (CLEO), pp. 1-1, 2015 Conference on, San Jose, CA, 2015.

4. H. Kalaycioğlu, K. Eken, and F. Ö. Ilday, "Fiber amplification of pulse bursts up to $20 \mu \mathrm{Jpulse}$ energy at $1 \mathrm{kHz}$ repetition rate," Opt. Lett., vol. 36, no. 17, p. 3383, 2011.

5. D. K. Kesim, H. Kalaycığlu, C. Kerse, and F. Ö. Ilday. "All-Fiber Burst Mode Femtosecond Laser System Integrated with OCT for Cataract Surgery," In The European Conference on Lasers and Electro-Optics, p. CL_5_1. Optical Society of America, 2015.

6. C. Kerse, H. Kalaycı̆ğlu, P. Elahi, Ö. Akaalan, and F. Ö. Ilday, "3.5-GHz intra-burst repetition rate ultrafast Yb-doped fiber laser," Opt. Commun., vol. 366, pp. 404-409, May 2016.

7. Weitkamp, Claus, ed. "Lidar: range-resolved optical remote sensing of the atmosphere," Springer Science \& Business, Vol. 102, 2006. 